\title{
Fenótipo neuropsicológico de crianças com síndrome de Down
}

Neuropsychology phenotype of children with Down syndrome

Fenotipo neuropsicológico de niños con síndrome de Down

\author{
Rosália Carmen de Lima Freire* \\ Nietsnie de Souza Duarte** \\ Izabel Hazin ${ }^{* * *}$
}

\begin{abstract}
Resumo
A síndrome de Down (SD) é a causa genética mais comum de deficiência intelectual, com incidência aproximada de um para cada 800 nascimentos vivos. A presença do cromossomo extra 21 (ou de regiôes críticas dele) associada a essa síndrome e a consequente alteração na dosagem gênica são responsáveis por diversas anormalidades estruturais e funcionais no sistema nervoso dessas crianças, que podem ser evidenciadas pelo atraso no desenvolvimento neuropsicomotor comumente observado. Alterações nos domínios da linguagem e memória são acentuadas e acabam dificultando a aprendizagem. Por outro lado, habilidades visuoespaciais apontadas como pontos fortes dessas crianças. Esses aspectos constituem um padrão de alterações e capacidades, característicos da SD, denominado fenótipo neuropsicológico. Neste artigo, será apresentada uma revisão de literatura das pesquisas que têm sido desenvolvidas acerca dos aspectos neuropsicológicos da SD e que vêm contribuindo para caracterizar o fenótipo neuropsicológico de crianças com essa síndrome, possibilitando intervençóes que focalizem áreas de potencial e minimizem áreas em defasagem.
\end{abstract}

Palavras-chave: Síndrome de Down. Fenótipo neuropsicológico. Avaliação neuropsicológica. Intervenção.

\section{Abstract}

Down syndrome (DS) is the most common genetic cause of intellectual disability, affecting approximately one for every 800 live births. The

\footnotetext{
*Mestranda em Psicologia pela Universidade Federal do Rio Grande do Norte. E-mail: rosaliacarmen@hotmail.com.

${ }^{* *}$ Mestranda em Psicologia pela Universidade Federal do Rio Grande do Norte. E-mail: nietsnieduarte@hotmail.com.

*** Doutora em Psicologia Cognitiva pela Universidade Federal de Pernambuco, professora adjunta III do Departamento de Psicologia da Universidade Federal do Rio Grande do Norte. E-mail: izabel.hazin@gmail.com.
} 
presence of extra chromosome 21 (or critical regions of it) associated with this syndrome, and the resultant change in gene dosage, is responsible for many structural and functional abnormalities in the nervous system of these children, which can be evidenced by psychomotor developmental delay commonly observed. Changes in the areas of language and memory are accentuated and end up hindering the learning process. On the other hand, visuospatial skills are seen as strengths of these children. The aspects mentioned above constitute a pattern of changes and skills characteristic of DS called neuropsychological phenotype. In this paper, we will presents a literature review of research that has been developed on the neuropsychological aspects of DS, and that has contributed to characterize the neuropsychological phenotype of children with this syndrome, allowing interventions that focus on areas of potential and minimize areas of weakness.

Keywords: Down syndrome. Neuropsychological phenotype. Neuropsychological evaluation. Intervention.

\section{Resumen}

El Síndrome de Down (SD) es la causa genética más común de discapacidad intelectual, que afecta aproximadamente a uno de cada 800 nacidos vivos. La presencia del cromosoma extra 21 (o de regiones críticas del mismo) asociados con este síndrome, y el consiguiente cambio en la dosis génica, es responsable de muchas anormalidades estructurales y funcionales en el sistema nervioso de estos niños, que pueden manifestarse por retraso en el desarrollo neuropsicomotor comúnmente observado. Cambios en las áreas de lenguaje y de la memoria se agudizan y terminan por afectar el aprendizaje. Por otro lado, las habilidades visoespaciales son vistas como puntos fuertes de estos niños. Los aspectos antes mencionados constituyen un patrón de cambios y capacidades característicos del SD denominado fenotipo neuropsicológico. En este trabajo, vamos a presentar una revisión bibliográfica de las investigaciones que se han desarrollado en los aspectos neuropsicológicos del SD, y que contribuyen a caracterizar el fenotipo neuropsicológico de niños con este síndrome, permitiendo las intervenciones que se centran en las áreas potenciales y minimicen las áreas desfasadas.

Palabras clave: Síndrome de Down. Fenotipo neuropsicológico. Evaluación neuropsicológica. Intervención. 


\section{Introdução}

A síndrome de Down (SD) foi primeiramente descrita há mais de cem $\triangle$ anos por John Lagdon Down e é considerada atualmente a causa genética mais comum de deficiência intelectual, com incidência aproximada de um para cada 700-1000 nascimentos vivos (Contestabile, Benfenati \& Gasparini, 2010; Tsao \& Kindelberger, 2009). Na maioria dos casos, essa síndrome está associada à “trissomia do 21 ”, que se refere à presença de três cromossomos 21, em vez de dois, em todas as células do organismo (Rondal, 1993; Silverman, 2007). Essa alteração na dosagem gênica, por sua vez, é responsável por uma série de comprometimentos no desenvolvimento neurológico dessas crianças evidenciados pelo atraso no desenvolvimento neuropsicomotor e presença de prejuízos variados em suas funções cognitivas (Schawartzman, 2003).

Alterações no domínio da linguagem são acentuadas nessa população e terminam por comprometer as trocas sociais (Martin, Klusek, Estigarribia \& Roberts, 2009). Além disso, dificuldades em manter a atenção e deficiências no campo da memória ocasionam prejuízos diversos na aprendizagem (Lott \& Dierssen, 2010). Por outro lado, do ponto de vista comportamental, são descritas como crianças alegres e sociáveis, embora se questionem atualmente esses estereótipos, já que também são observados comportamentos problemáticos, tais como teimosia, ritualização e impulsividade (Wuo, 2007).

Esses aspectos constituem um padrão de alterações cognitivas, comportamentais, sociais e afetivas característicos da SD, aqui denominado fenótipo neuropsicológico. Ressalta-se que estudos vêm sendo realizados com o intuito de estabelecer fenótipos neuropsicológicos ou comportamentais associados a quadros clínicos específicos. Esses estudos têm como objetivo realizar uma descrição do perfil de capacidades desses indivíduos, apontando as suas áreas de forças e de fraquezas (Silverman, 2007). Dessa forma, eles se afastam das pesquisas que buscaram identificar apenas deficiências no desenvolvimento dessas crianças, e procuram, por sua vez, realizar uma análise minuciosa de seus perfis neuropsicológicos por meio de comparações com outras síndromes genéticas, ou entre a SD e crianças com desenvolvimento típico (DT) (Tsao \& Kindelberger, 2009).

O conceito de fenótipo neuropsicológico da SD implica a defesa da existência de um perfil de desenvolvimento específico para essa população (Silverman, 2007). Esse perfil, que é provavelmente atribuível a uma 
superexpressão de um conjunto de genes que residem no cromossomo 21, inclui, de maneira geral, pontos fortes em determinadas habilidades, tais como as visuoespaciais, bem como déficits em outros conjuntos, como as habilidades de linguagem expressiva, memória verbal, memória explícita, entre outros (Kogan et al., 2009; Menghini, Contanzo \& Vicari, 2011; Tsao \& Kindelberger, 2009; Wang, 1996).

No entanto, quando se faz referência ao fenótipo neuropsicológico da $\mathrm{SD}$, não significa que se pressupõe a existência um padrão estereotipado e previsível de desenvolvimento para todas as crianças que têm essa síndrome. Isso porque, como se sabe, o desenvolvimento neuropsicológico não depende exclusivamente das alteraçôes cromossômicas que são observadas, mas também do restante do potencial genético e, de modo fundamental, das importantes influências do ambiente sócio-histórico-cultural no qual a criança está inserida (Schawartzman, 2003).

Ao se buscar caracterizar o fenótipo neuropsicológico da $S D$, não se pretende, portanto, massificar quadros clínicos nem tampouco rotular prognósticos, mas oferecer informações que estimulem a reflexão sobre as dificuldades escolares e sociais mais apresentadas por essas crianças, bem como as habilidades que elas desempenham satisfatoriamente (Schawartzman, 2003). Ressalta-se que, com base nesse conhecimento, será possível o desenvolvimento de estratégias de intervenção, neuropsicológica e educacional, cada vez mais eficazes.

Nesse sentido, este artigo tem como objetivo apresentar uma revisão das pesquisas que têm sido desenvolvidas acerca dos aspectos neu ropsicológicos da SD e que vêm contribuindo com isso para caracterizar o fenótipo neuropsicológico de crianças com essa síndrome. Serão discutidos os principais achados neuropsicológicos encontrados em diferentes domínios, tais como nível intelectual, linguagem, memória, atenção, funçôes executivas, assim como aspectos comportamentais e socioafetivos de crianças com SD.

\section{Método}

Foi realizada uma revisão de literatura, por meio da busca de referências em bases de dados eletrônicas (Pubmed, Science Direct, Scielo e Lilacs), utilizando-se da combinação dos seguintes descritores: Down syndrome, neuropsychological, memory, attention, executive functions, language, cognitive phenotype, behavioral phenotype. Foram selecionados artigos originais e de revisão que obedeciam aos seguintes critérios de inclusão: 
a) faixa etária: prioritariamente de 0 a 18 anos. Cabe ressaltar que foram incluídos nesta revisão cinco artigos que utilizaram amostras de adultos com SD, pois, apesar de não ser o foco desta revisão, considerou-se que os textos contribuíam de maneira significativa para a melhor compreensão do tema; b) estudos publicados nos últimos dez anos; c) artigos escritos no idioma inglês, português, espanhol ou francês; d) foco em aspectos neuropsicológicos; e) e artigos disponíveis na íntegra.

Os artigos selecionados foram lidos e analisados a fim de determinar a sua relevância para esta revisão, seguindo-se da separação destes de acordo com a função neuropsicológica investigada. A seguir, serão discutidos os principais achados dos estudos incluídos nesta revisão.

\section{Fenótipo neuropsicológico de crianças com SD}

\subsection{Nível intelectual}

A deficiência intelectual tem sido descrita como uma das características mais marcantes do fenótipo neuropsicológico da SD. Geralmente, o nível intelectivo dessas crianças (QI) situa-se na faixa classificada entre moderada e grave retardo mental (QI = 25-55) (Tsao \& Kindelberger, 2009; Vicari, 2006). No entanto, é importante frisar a grande variabilidade encontrada nos resultados de QI dessas crianças, podendo oscilar entre 50 até 60 pontos entre os diferentes indivíduos (Turner \& Alborz, 2003).

Um dado que merece atenção e que precisa ser discutido, a fim de se evitarem interpretações errôneas, refere-se ao aparente declínio no QI que pode ser observado conforme as crianças com SD vão envelhecendo. De acordo com Schawartzman (2003), esse declínio pode ser resultado da forma como o QI tem sido avaliado. Isso porque, nessas avaliações, as crianças e adolescentes com SD são comparadas a crianças e adolescentes de desenvolvimento típico (DT) da mesma idade. Tal metodologia não se apresenta, portanto, como a mais indicada, visto que é necessário que se leve em consideração que o desenvolvimento na SD é mais lento, o que erroneamente vem sendo interpretado como rebaixamento da capacidade intelectiva. Esse tipo de comparação, segundo o mesmo autor, acaba desprezando importantes ganhos que os indivíduos com SD podem estar apresentando em vários aspectos de seu funcionamento cognitivo.

Outro ponto que merece destaque e que vem sendo investigado diz respeito ao efeito da estimulação precoce sobre o desenvolvimento de 
crianças com SD, implicando um aumento de seus QIs. Em um estudo antigo, porém clássico, Ludlow \& Allen (1979) encontraram diferenças significativas entre dois grupos de crianças com SD estudados (um que participava de programas de estimulação e outro não), favorecendo o desenvolvimento das crianças estimuladas. Eles verificaram um QI médio de 82,7 no grupo estimulado e 66,4 nos controles. Além disso, as crianças estimuladas foram mais facilmente integradas na escola, e o acompanhamento escolar em anos posteriores constatou que uma proporção considerável continuou os estudos em escolas regulares (40\% em detrimento dos $29 \%$ no grupo controle).

\subsection{Linguagem}

O campo da linguagem tem sido amplamente descrito como uma das áreas que mais apresentam prejuízos na SD. Embora o comportamento vocal pré-linguístico nos bebês desenvolva-se na faixa considerada de normalidade, foram encontrados graves déficits no desenvolvimento da linguagem em crianças com SD no que diz respeito a aspectos fonológicos e sintáticos (Martin, Klusek, Estigarribia \& Roberts, 2009). Conforme atenta Silverman (2007), padrões de comunicação qualitativamente atípicos estão associados à SD a partir de uma tenra idade, sendo encontrados diversos níveis de desenvolvimento de habilidades linguísticas abaixo do que é observado em crianças de DT com a mesma idade mental (Contestabile, Benfenati \& Gasparini, 2010).

A gravidade de comprometimento da linguagem é altamente variável, estando os diversos componentes do sistema linguístico afetados em graus diferentes. Em geral, a linguagem expressiva encontra-se mais prejudicada quando comparada à linguagem receptiva e (ou) à compreensão da linguagem (Ypsilanti \& Grouios, 2008; Lanfranchi, Jerman \& Vianello, 2009; Menghini, Contanzo \& Vicari, 2011; Rondal \& Comblain, 1996; Silverman, 2007). Estudos sugerem que o vocabulário receptivo é um dos pontos fortes do funcionamento cognitivo desse grupo, enquanto o desenvolvimento do processamento sintático e morfossintático, por outro lado, encontra-se particularmente afetado (Cleland, Wood, Hardcastle, Wishart \& Timmins, 2010; Silverman, 2007).

Normalmente, em crianças com SD, a emissão das primeiras palavras ocorre em torno dos 18 meses de idade (o que significa um atraso de quatro meses em relação a crianças de DT). Segundo Marder e Cholmáin (2006), como essas crianças ao longo do seu desenvolvimento linguístico 
apresentam maiores dificuldades em expressar-se (dificuldades caracterizadas por uma menor clareza de seus discursos e uso simplificado da gramática), isso acaba, muitas vezes, mascarando a força relativa que elas têm na compreensão da linguagem.

Crianças com SD têm um perfil anatômico específico que pode afetar a produção da fala (Cleland, Wood, Hardcastle, Wishart \& Timmins, 2010). A capacidade de criar as articulações necessárias para a elaboração do discurso, por exemplo, é prejudicada pela menor cavidade bucal (que frequentemente dá uma impressão de uma língua grande). Além disso, a hipotonia dos músculos ao redor da boca e dificuldades do controle motor na produção da fala também dificultam a capacidade de comunicação desses indivíduos (Marder \& Cholmáin, 2006; Martin, Klusek, Estigarribia \& Roberts, 2009).

As perdas auditivas, que ocorrem em cerca de dois terços das crianças com $\mathrm{SD}$, também têm sido associadas às dificuldades de linguagem encontradas nessas crianças (Martin, Klusek, Estigarribia \& Roberts, 2009; Roberts, Price \& Malkin, 2007). Quando presentes, essas perdas podem dificultar a aquisição da linguagem e o desenvolvimento intelectual, sendo, portanto, imprescindível o seu devido tratamento. No entanto, não se pode afirmar que essas perdas determinam as dificuldades na linguagem, já que não existem provas definitivas que o prejuízo nessa área seja consequência da audição diminuída (Vicari, 2006).

Pesquisas vêm indicando que crianças com SD apresentam preferência e empregam mais o meio comunicativo gestual em detrimento do meio verbal, devido às suas dificuldades na linguagem oral (Almeida \& Limongi, 2010; Cunha \& Limongi, 2008; Ypsilanti \& Grouios, 2008; Vicari, 2006). Os gestos têm um papel importante, já que funcionam não apenas como elementos de transição de ações motoras para a linguagem falada, mas também como facilitadores do processo de produção da fala. Eles fornecem à criança recursos cognitivos extras que permitem que elas representem e comuniquem ideias mais complexas quando ainda não foram capazes de fazê-las por meio oral (Almeida \& Limongi, 2010).

Nas crianças com SD, os gestos teriam uma função social importante, já que ofereceriam a estas maiores possibilidades de interação. Nessas crianças, o uso dos gestos como principal meio de comunicação ocorre durante longos períodos, visto que a expressão verbal dos seus significados é dificultada por uma série de fatores (déficits na memória verbal, da motricidade fina, etc.). Tem sido observado também que, durante o período de expansão do vocabulário, as crianças com $\mathrm{SD}$, em vez de substituírem progressivamente 
os gestos pelas palavras correspondentes em suas produçôes, usam os gestos simultaneamente às palavras ou ainda usam as palavras como suporte aos gestos, e não o contrário, como comumente ocorre em crianças de DT (Almeida \& Limongi, 2010).

Apesar das deficiências de linguagem e cognitivas que as crianças com SD apresentam, muitas delas conseguem alfabetizar-se (Cleland, Wood, Hardcastle, Wishart \& Timmins, 2010). Para que elas consigam adquirir cada vez mais habilidades, é imprescindível que os professores e outros profissionais que atuam junto a essas crianças obtenham um melhor conhecimento acerca de seus perfis cognitivos, a fim de melhor orientar as suas práticas de intervenção.

\subsection{Memória}

A memória é uma das funções neuropsicológicas mais complexas e tem sido descrita e classificada de maneiras diversas ao longo dos anos. Estudos com pacientes que sofreram lesões cerebrais e com indivíduos normais forneceram evidências de que o sistema de memória não é unitário, mas pode ser dividido em diferentes componentes, que são mediados e conduzidos por circuitos neurais distintos (Baddeley, 1992; Malloy-Diniz, Fuentes, Mattos \& Abreu, 2010). Uma das classificações propostas usa a categoria tempo para descrever os sistemas de memória, originando os sistemas específicos de memória de "curto prazo" e um armazenamento de memória de longo prazo. Além disso, os sistemas de memória de curto e de longo prazo também podem ser subdivididos com base na classificação pautada pela natureza do material armazenado (Jarrold, Nadel \& Vicari, 2008).

Em relação à memória de longo prazo, estudos indicam que crianças com SD apresentam um desempenho significativamente inferior em tarefas de memória explícita quando comparados a crianças com DT. Em uma pesquisa que comparou esse domínio em crianças com SD com indivíduos com deficiência intelectual não especificada e crianças de DT com a mesma idade mental, o grupo com SD obteve uma pontuação significativamente menor do que a dos outros dois grupos nos testes de lista de palavras e história curta, assim como na reprodução por recordação da figura complexa de Rey (Carlesimo, Marotta \& Vicari, 1997; Jarold, Nadel \& Vicari, 2008).

No entanto, em relação a tarefas que requerem processamento da memória implícita, crianças com SD apresentam um desempenho considerado normal (Contestabile, Benfenati \& Gasparini, 2010; Jarold, Nadel \& Vicari, 2008; Vicari, 2006), o que indica uma dissociação funcional 
entre esses dois mecanismos de memória. De fato, a memória implícita é sustentada substancialmente por processos automáticos que requerem baixa atenção, enquanto a memória explícita lida com a aprendizagem consciente e intencional e requer codificação de informação, estratégias de recuperação e elevado grau de atenção. Dessa forma, a codificação de informações pobre, habilidade de recuperação prejudicada, assim como déficits de atenção, todos eles identificados na SD, podem ser responsáveis pelo comprometimento seletivo da memória explícita que é observado em crianças com essa síndrome (Contestabile, Benfenati \& Gasparini, 2010).

Como já mencionado, muitos modelos têm sido propostos para explicar os processos de memória. Baddeley e Hitch (1974), por exemplo, propuseram um modelo para a memória de curto prazo que parece ser particularmente propício para a discussão dos prejuízos de memória que são associados com a SD, a saber, a memória operacional (ou de trabalho). Este foi apresentado como sendo o sistema responsável pelo armazenamento temporário e manipulação de informações necessárias para o desempenho em uma ampla gama de habilidades cognitivas, tais como a aprendizagem, leitura, escrita, processamento linguístico, orientação, compreensão, raciocínio e processamento aritmético (Lanfranchi, Carretti, Spanò \& Cornoldi, 2009; Lanfranchi, Jerman \& Vianello, 2009; Silverman, 2007).

A memória operacional pode ser dividida em diferentes subsistemas, responsáveis por tarefas distintas, porém relacionadas. O primeiro sistema corresponde ao sistema supervisor, o executivo central, que é responsável pela coordenação do fluxo de informações, pelas estratégias de recuperação de traços da memória de longo prazo e pelo raciocínio lógico e aritmético. Dois outros sistemas subsidiários ou "escravos" seriam a alça fonológica e o esboço visuoespacial (Baddeley e Hitch, 1974; Baddeley, 1992). A alça fonológica é responsável pelo armazenamento temporário de informações verbais, de duração limitada, e que são mantidos através do processo de articulação. $\mathrm{O}$ esboço visuoespacial, por outro lado, é responsável pelo armazenamento de informaçôes visuoespaciais ao longo de períodos breves e desempenha um papel fundamental na geração e manipulação de imagens mentais (Lanfranchi, Jerman, Vianello, 2009; Lanfranchi, Carretti, Spanò \& Cornoldi, 2009).

Os estudos que analisaram a memória operacional na SD têm apontado para a presença de déficits no seu componente verbal, enquanto que o esboço visuoespacial encontra-se relativamente preservado (Carretti \& Lanfranchi, 2010; Duarte, Covre, Braga \& Macedo, 2011; Jarrold, Nadel \& Vicari, 2008; Lanfranchi, Jerman \& Vianello, 2009; Laws, 2002; Silverman, 2007). Em relação a esses déficits, alguns pesquisadores vêm propondo que o baixo 
desempenho de crianças com SD reflete as dificuldades generalizadas que essas crianças têm em tarefas que requerem um processamento verbal, sendo resultado de suas capacidades linguísticas diminuídas. Porém, segundo Lanfranchi, Jerman \& Vianello (2009), a direção da relação poderia ser o oposto, ou seja, os déficits na memória verbal afetariam a aquisição das habilidades linguísticas na SD.

Lanfranchi, Jerman \& Vianello (2009) investigaram a relação entre o baixo desempenho de crianças com SD em tarefas de memória verbal e a presença de déficits nas habilidades linguísticas, notadamente buscando responder se estas se deviam a um déficit específico no sistema de memória verbal desses indivíduos. Os resultados desse estudo, semelhantes aos de Laws (2002), demonstraram que o déficit na memória de trabalho verbal encontrado na SD não pode ser atribuído simplesmente ao comprometimento da linguagem, já que, quando comparados com crianças de DT e com o mesmo nível de vocabulário ou capacidade verbal, crianças com $\mathrm{SD}$, ainda assim, apresentam um menor desempenho em todas as tarefas de memória verbal.

As diferenças encontradas têm sido atribuídas a algum tipo de déficit específico na alça fonológica de crianças com SD, podendo refletir, por exemplo, uma redução na capacidade de armazenamento. Assim, tanto a memória de trabalho verbal quanto as habilidades de linguagem poderiam ser prejudicadas de formas independentes na SD (Lanfranchi, Jerman \& Vianello, 2009; Jarrold, Nadel \& Vicari, 2008).

Por outro lado, diversas pesquisas têm sugerido que a memória visuoespacial encontra-se relativamente preservada em crianças com SD, relatando-se prejuízos em apenas alguns de seus aspectos. Atualmente, considera-se que a memória de trabalho visuoespacial pode ser dividida em um componente espacial (envolvido na memória de posições) e um componente visual (envolvido na memória de objetos e suas propriedades). Segundo Jarrold, Nadel \& Vicari (2008), apesar de crianças com SD apresentarem um melhor desempenho em tarefas visuoespaciais do que em tarefas de memória verbal, quando essas tarefas visuoespaciais são dissociadas, crianças com SD se saem melhor se a tarefa envolver apenas o aprendizado de uma sequência espacial do que na memorização de objetos visuais.

\subsection{Atenção}

A atenção é considerada a base para a organização dos processos mentais, pois confere a estes diretividade, seletividade e estabilidade. Ela representa a condição inicial e decisiva para as funções cognitivas em sua totalidade, 
particularmente para o processo de aprendizagem e de memorização. Indivíduos com déficit de atenção, em geral, não apresentam bom desempenho nesses processos (Macêdo, Lima, Cardoso \& Beresford, 2009). Uma das modalidades da atenção, a atenção sustentada, está relacionada à capacidade de detectar eventos imprevisíveis e raros durante períodos prolongados de tempo, e inclui a vigilância e o estado de alerta. Relatos neuroanatômicos sugerem que áreas pré-frontais, particularmente o córtex pré-frontal direito, são mais ativadas durante tarefas de vigilância. Além dessas áreas, lesões no córtex frontal e no corpo caloso também têm sido relacionadas a deficiências no desempenho em testes de atenção sustentada. Evidências de uma associação entre lesões nessas áreas e déficits na atenção são de interesse para os pesquisadores que trabalham com a SD, visto que regiões frontais, temporais e o corpo caloso já foram identificados como apresentando subdesenvolvimento na SD (Trezise, Gray \& Sheppard, 2008).

Quando investigada em estudos com crianças com SD (Brown et al., 2003), a atenção sustentada têm sido relatada como sendo menos eficaz nessas crianças se comparadas com crianças de DT. O estudo de Trezise, Gray \& Sheppard (2008), por exemplo, procurou investigar a hipótese de que crianças com SD demonstram um desempenho superior em tarefas de atenção sustentada que utilizam como recurso material visual (no caso do estudo, os estímulos - figuras de animais - eram apresentados na tela de um computador) do que em tarefas que utilizam material auditivo (nomes dos animais eram emitidos pelo alto-falante conectado ao computador). Os resultados encontrados apontaram para um melhor desempenho nas tarefas de atenção sustentada cuja modalidade de apresentação foi visual, o que sugere que crianças com SD podem se beneficiar da apresentação de um material educativo que use o meio visual, já que este facilita a manutenção da atenção e aprendizagem.

Considera-se que o déficit de atenção que é observado em crianças com SD interfere negativamente no seu desenvolvimento, já que dificulta a iniciação, organização e, principalmente, a manutenção do envolvimento na realização de determinadas tarefas, necessárias para a aprendizagem destas crianças (Macêdo, Lima, Cardoso \& Beresford, 2009).

\subsection{Funçõos executivas}

As funçôes executivas (FE) se referem a um conjunto de habilidades cognitivas inter-relacionadas que permitem aos indivíduos engajarem-se voluntariamente em comportamentos orientados a objetivos. Tais funções são fundamentais ao direcionamento e regulação de várias habilidades 
intelectuais, emocionais e sociais, e são associadas com o funcionamento da parte frontal do cérebro, embora se reconheça que diferentes áreas também podem estar envolvidas (Dias, Menezes \& Seabra, 2010; Lanfranchi, Jerman, Dal Pont, Alberti \& Vianello, 2010).

Habilidades de FE são úteis para a resolução de problemas, formação de conceitos, alternância de tarefas, iniciação, planejamento, controle de impulsos, efetivação das ações, flexibilidade cognitiva e comportamental, e monitoramento das atitudes. De forma agrupada, esses processos cognitivos permitem ao indivíduo iniciar, planejar, sequenciar e monitorar seus comportamentos e açōes (Dias, Menezes \& Seabra, 2010; Lanfranchi, Jerman, Dal Pont, Albetti \& Vianello, 2010).

Recentemente, estudos têm sido realizados com o intuito de investigar as FE na SD. A maioria desses estudos incluiu amostras de adultos (Adams \& Oliver, 2010; Rowe, Lavender \& Turk, 2006), propondo a presença de déficits nessa área. No entanto, de acordo com Lanfranchi, Jerman, Dal Pont, Albetti \& Vianello (2010), ainda é escasso o exame do funcionamento executivo de adolescentes e crianças com SD, e os poucos estudos que foram realizados fornecem resultados contraditórios. Assim, pesquisas ainda são necessárias para explorar FE nesse grupo clínico, a fim de determinar as causas dos déficits nas FE nessa população: se os déficits nas FE encontrados na amostra de adultos estão relacionados com o declínio cognitivo associado com a idade e (ou) doença de Alzheimer ou se são uma característica do fenótipo cognitivo dessa síndrome.

Lanfranchi, Jerman, Dal Pont, Albetti \& Vianello (2010) investigaram as FE em adolescentes com SD, aplicando nessa amostra e em crianças de DT, pareadas por idade mental, uma vasta bateria de testes neuropsicológicos (Stroop, torre de Londres, fluência verbal, entre outros). Os resultados desse estudo demonstraram a presença de um déficit no funcionamento executivo dos adolescentes sindrômicos, com prejuízos sobretudo nas tarefas de flexibilidade, planejamento, resolução de problemas, memória de trabalho e inibição. No entanto, os resultados desse estudo sugerem também que nem todos os processos de FE são prejudicados na $\mathrm{SD}$, necessitando-se assim a realização de mais estudos que possam compreender melhor quais tarefas são mais prejudicadas e quais se encontram relativamente preservadas.

\subsection{Aspectos comportamentais e socioafetivos do fenótipo neuropsicológico de crianças com SD}

Apesar das dificuldades relatadas na comunicação oral de crianças com SD, muitas delas apresentam um funcionamento social considerado 
adequado. Foi observado que essas crianças relacionam-se bem com seus pares, mostrando-se mais empáticas do que crianças com outros tipos de deficiências. Estudos como o realizado por Fidler (2005) destacam que essas crianças podem até usar sua força relativa nas habilidades sociais para compensar deficiências em outros domínios. Em um estudo no qual se utilizaram com crianças com SD tarefas consideradas muito difíceis, observou-se que estas não apenas estabeleciam mais contatos visuais com experimentadores durante o momento do teste como também "comportamentos encantadores" que envolviam o experimentador socialmente (Fidler, 2005; Fidler, Most \& Philofsky, 2008).

Crianças com SD também podem enviar mais sinais emocionais positivos do que outras crianças com deficiência intelectual. Em um estudo com crianças com SD, na faixa etária entre 5 e 12 anos de idade, observouse que estas sorriam com mais frequência do que crianças com outros tipos de deficiência intelectual, embora esse achado tenha mudado com indivíduos com SD que se aproximavam da idade adulta (Fidler, 2005). Frequentemente, essas crianças são descritas como tendo personalidades encantadoras, de acordo com um estereótipo de personalidade positiva associada a essa síndrome. Em um estudo de Caar (1995), mais de 50\% das crianças estudadas com SD foram descritas como "afetuosas", "boas", "amáveis", e muitas também foram descritas como "alegres" "generosas" e "divertidas".

Ao lado dessas frequentes descrições positivas, muitas crianças com SD também são descritas como apresentando inconsistências em suas orientações motivacionais. Elas podem mostrar níveis mais baixos de persistência e níveis mais elevados de distração durante execução de tarefas, o que comumente interfere na conclusão destas. Tais crianças são, às vezes, descritas como teimosas, sem força de vontade, características que podem contribuir para a recusa ao finalizar essas tarefas (Fidler, 2005).

Outros problemas comportamentais e de atenção também têm sido relatados pelos pais de crianças e adolescentes com SD. Fidler (2005), ao relatar estudos que investigaram manifestações comportamentais em crianças com SD, destaca que, quando comparadas com irmãos sem a síndrome, crianças com SD apresentavam uma hiperatividade maior; no entanto, em relação a comportamentos depressivos e ansiosos, as pontuações não diferiram significativamente entre os dois grupos, exceto em amostras de adolescentes e adultos com a síndrome, nos quais era maior a ocorrência de transtornos psiquiátricos, tais como a depressão. No geral, ressalta-se que, apesar dos estudos relatarem mais problemas 
de comportamento no grupo de crianças com SD, em comparação aos seus irmãos, crianças com SD não chegaram a atingir níveis considerados indicativos de comportamentos desviantes.

\section{Caracterização do fenótipo neuropsicológico e intervenção na SD}

Nos últimos anos, pesquisas neuropsicológicas têm contribuído para maior compreensão e identificação de diferentes fenótipos neuropsicológicos entre os indivíduos com deficiência intelectual. O perfil da SD, por exemplo, tem sido caracterizado por dificuldades na área da linguagem e habilidades visuoespaciais relativamente preservadas, o que contrasta com o perfil de outra síndrome bastante estudada, a síndrome de Willians, na qual se observa um padrão de dificuldades em certas habilidades visuoespaciais e relativa preservação da linguagem produtiva e receptiva (Vicari, 2006).

Os estudos supracitados fazem parte de abordagem teórica que considera a deficiência intelectual não apenas como simples atraso no desenvolvimento cognitivo normal, mas sim como perfis característicos, que podem ser qualitativamente especificados. Esse ponto de vista teórico sugere a necessidade de protocolos de intervenção individualizados, baseados em estudos que apontam não só as habilidades cognitivas prejudicadas, mas, não menos importante, os respectivos pontos fortes e as habilidades relativamente preservadas em crianças com SD (Vicari, 2006).

Partindo desse ponto de vista, este artigo procurou expor os principais achados neuropsicológicos encontrados em crianças com SD, explicitando as áreas que apresentam maiores dificuldades, bem como habilidades preservadas nessa síndrome.

Silva e Kleinhans (2006) observam que as descobertas em relação ao fenótipo cognitivo (ou neuropsicológico) da SD são determinantes na organização do trabalho a ser desenvolvido pelos diversos profissionais. Apenas com a compreensão desse fenótipo, será possível o estabelecimento de intervenções que focalizem áreas de potencial e minimizem áreas em defasagem. Na maioria das intervenções que têm sido realizadas, é feito o contrário, pois são trabalhados somente os déficits característicos do fenótipo. Sugere-se, então, a importância da compreensão do fenótipo neuropsicológico, notadamente em termos da identificação de forças, bem como das estratégias compensatórias desenvolvidas que promovem adaptação e consequentemente maior qualidade de vida. 
A plasticidade cerebral nos ajuda a compreender como um trabalho adequado de estimulação que focalize aspectos do fenótipo neuropsicológico pode contribuir com desenvolvimento da criança com SD. Entende-se por plasticidade cerebral a capacidade adaptativa do sistema nervoso central de modificar sua organização estrutural e funcional (Kandel, Schuwartz \& Jessell, 2003; Silva \& Kleinhans, 2006). Já se sabe que essa reorganização cerebral é diretamente influenciada pelas interações que são realizadas com o ambiente externo. Isso porque, a cada nova experiência, novas ligações entre os neurônios podem surgir, ser reordenadas, possibilitando, assim, a base do aprendizado e da memória (Ferreira, Ferreira \& Oliveira, 2010).

\section{Considerações finais}

Como bem ressaltava Vygotsky (1997), crianças cujo desenvolvimento é atravessado por algum tipo de deficiência não são simplesmente menos desenvolvidas do que seus pares "normais"; essas crianças se desenvolvem de modo qualitativamente diferente. Embora a deficiência imponha algumas limitações e dificuldades, ela também pode impulsionar o indivíduo para a compensação. O estudo da pessoa com algum tipo de deficiência não deveria centrar-se, portanto, apenas na determinação do nível e gravidade da deficiência, mas levar em consideração os processos compensatórios e substitutivos adotados pelo indivíduo no seu contexto de desenvolvimento (Vygotsky, 1997; Duarte, Freire \& Hazin, 2012).

O desenvolvimento de uma criança atravessado por uma deficiência caracteriza-se como processo de criação e recriação, fundado na reorganização de todas as funções, na formação de novos processos substitutivos e abertura de caminhos alternativos para o desenvolvimento. Dessa forma, é de suma importância que os profissionais que trabalham junto com essas crianças conheçam bem as suas peculiaridades, e aqui se destacam as características do seu fenótipo neuropsicológico e (ou) comportamental, para assim melhor adequar os seus métodos de trabalho com essas crianças.

Como discutido ao longo deste artigo, observa-se que o funcionamento neuropsicológico da SD é caracterizado pela presença de múltiplas áreas afetadas. No entanto, os conceitos de plasticidade sugerem que podemos criar novas rotas neuronais e novas estratégias cognitivas que possibilitem a minimização e (ou) superação dos déficits apresentados. Nesse sentido, aponta-se para a necessidade de realização de novos 
estudos que contribuam para o avanço no estabelecimento do fenótipo neuropsicológico de crianças com SD, assim como de outros grupos clínicos, potencializando suas habilidades e promovendo a aprendizagem e desenvolvimento dessas crianças.

\section{Referências}

Adams, D. \& Oliver, C. (2010). The relationshipbetween acquired impairments of executive function and behaviour change in adults with Down syndrome. Journal of Intellectual Disability Research, 54 (5), 393-405.

Almeida, F. C. F. \& Limongi, S. C. O. (2010). O papel dos gestos no desenvolvimento da linguagem oral de crianças com desenvolvimento típico e crianças com síndrome de Down. Revista da Sociedade Brasileira de Fonoaudiologia, 15 (3), 458-464.

Baddeley, A. (1992). Working Memory. Science, 255, 556-559.

Baddeley, A. D. \& Hitch, G. J. (1974). Working memory. In: G. Bower (ed.). The psychology of learning and motivation. New York: Academic Press.

Brown, J. H., Johnson, M. H., Paterson, S. J., Gilmore, R., Longhi, E. \& Karmiloff-Smith, A. (2003). Spatial representation and attention in toddlers with Williams syndrome and Down syndrome. Neuropsychologia, 41 (8), 1037-1046.

Carlesimo, G. A., Marotta, L. \& Vicari, S. (1997). Long-term memory in mental retardation: Evidence for a specific impairment in subjects with Down's syndrome. Neuropsychologia, 35 (1), 71-79.

Carr, J. (1995). Down's Syndrome: children growing up. Cambridge: Cambridge University Press.

Carretti, B. \& Lanfranchi, S. (2010). The effect of configuration on VSWM performance of Down syndrome individuals. Journal of Intellectual Disability Research, 54 (12), 1058-1066.

Cleland, J., Wood, S., Hardcastle, W. J., Wishart, J. G. \& Timmins, C. (2010). The relationship between speech, oromotor, language and cognitive abilities in children with Down's syndrome. International Journal of Language and Communication Disorders, 45 (1), 83-95. 
Contestabile, A., Benfenati, F. \& Gasparini, L. (2010). Communication breaks-Down: From neurodevelopment defects to cognitive disabilities in Down syndrome. Progress in Neurobiology, 91 (1), 1-22.

Cunha, E. P. \& Limongi, S. C. O. (2008). Modo comunicativo utilizado por crianças com síndrome de Down. Pró-Fono Revista de Atualização Científica, 20 (4), 243-248.

Dias, N. M., Menezes, A. \& Seabra, A. G. (2010). Alterações das funções executivas em crianças e adolescentes. Estudos Interdisciplinares em Psicologia, 1 (1), 80-95.

Duarte, C. P., Covre, P., Braga, A. C. \& Macedo, E. C. (2011). Visuospatial support for verbal short-term memory in individuals with Down syndrome. Research in developmental disabilities, 32 (5), 1918-1923.

Duarte, N. S., Freire, R. C. L., Hazin, I. (2012). Notas sobre aspectos epistemológicos e históricos da Psicologia histórico-cultural. Memorandum, $22,52-67$.

Ferreira, D. R. S. A., Ferreira, W. A. \& Oliveira, M. S. (2010). Pensamento e linguagem em crianças com síndrome de Down: um estudo de caso da concepção das professoras. Ciências e Cognição, 15 (2), 216-227.

Fidler, D. J. (2005). The emerging Down syndrome behavioral phenotype in early childhood: implications for practice. Infants \& Young Children, 18 (2), 86-103.

Fidler, D. J., Most, D. E., \& Philofsky, A. D. (2008). The Down syndrome behavioural phenotype: taking a developmental approach. Down Syndrome Research and Practice, 12 (3), 188-195.

Jarrold, C., Nadel, L. \& Vicari, S. (2008). Memory and neuropsychology in Down syndrome. Down Syndrome Research and Practice, 12 (3), 68-73.

Kandel, E. R., Schuwartz, J. H. \& Jessell, T. M. (2003). Principios da Neurociência. (4 ed). Barueri: Manole.

Kogan, C. S. et al. (2009). A comparative neuropsychological test battery differentiates cognitive signatures of Fragile $\mathrm{X}$ and Down syndrome. Journal of Intellectual Disability Research, 53 (2), 125-142.

Lanfranchi, S., Carretti, B., Spanò, G. \& Cornoldi, C. (2009). A specific deficit in visuospatial simultaneous working memory in Down syndrome. Journal of Intellectual Disability Research, 53 (5), 474-483.

Lanfranchi, S. Jerman, O. \& Vianello, R. (2009). Working memory and cognitive skills in individuals with Down syndrome. Child Neuropsychology, 15 (4), 397-416. 
Lanfranchi, S., Jerman, O., Dal Pont, E., Albetti, A. \& Vianello, R. (2010). Executive function in adolescents with Down syndrome. Journal of Intellectual Disability Research, 54 (4), 308-319.

Laws, G. (2002). Working memory in children and adolescents with Down syndrome: evidence from a colour memory experiment. Journal of Child Psychology and Psychiatry, 43 (3), 353-364.

Lott, I. T. \& Dierssen, M. (2010). Cognitive deficits and associated neurological complications in individuals with Down's syndrome. Lancet Neurol, 9 (6), 623-633.

Ludlow, J. R. \& Allen, L. M. (1979). The effects of early intervention and preschool stimulus on the development of the Down's syndrome child. Journal of Mental Deficiency Research, 23, 29-44.

Macêdo, L., Lima, I., Cardoso, F. \& Beresford, H. (2009). Avaliação da relação entre o déficit de atenção e o desempenho grafomotor em estudantes com síndrome de Down. Revista Brasileira de Educação, 15 (3), 431-440.

Malloy-Diniz, L. F., Fuentes, D., Mattos, P. \& Abreu. N (org.). (2010). Avaliação Neuropsicológica. Porto Alegre: Artmed.

Marder, L. \& Cholmáin, C. N. (2006). Promoting language development for children with Down's syndrome. Current Paedriatics, 16 (7), 495-500.

Martin, G., Klusek, J., Estigarribia, B. \& Roberts, J. (2009). Language characteristics of individuals with Down syndrome. Topics in Language Disorders, 29 (2), 112-132.

Menghini, D., Contanzo, F. \& Vicari, S. (2011). Relationship between brain and cognitive processes in down syndrome. Behavior Genetics, 41 (3), 381-393.

Roberts, J. E., Price, J. \& Malkin, C. (2007). Language and communication development in down syndrome. Mental Retardation and Developmental Disabilities Research Reviews, 3 (1), 26-35.

Rondal, J. A. (1993). Down's syndrome. In: Bishop, D. \& Mogford, K. (orgs.). Language development in exceptional circumstances. Hillsdale: Laurence Erlbaum.

Rondal, J. A. \& Comblain, A. (1996). Language in adults with Down syndrome. Down Syndrome Research and Practice, 4 (1), 3-14.

Rowe, J., Lavender, A. \& Turk, V. (2006). Cognitive executive function in 
Down's syndrome. British Journal of Clinical Psychology, 45 (1), 5-17.

Schawartzman, J. S. (org.). (2003). Síndrome de Down. São Paulo: Memnon.

Silva, M. F. M. C. \& Kleinhans, A. C. S. (2006). Processos cognitivos e plasticidade cerebral na Síndrome de Down. Revista Brasileira de Educação Especial, 12 (1), 123-138.

Silverman, W. (2007). Down syndrome: cognitive phenotype. Mental Retardation and Developmental Disabilities Research Reviews, 13 (3), 228-236.

Trezise, K. L., Gray, K. M. \& Sheppard, D. M. (2008). Attention and vigilance in children with Down syndrome. Journal of Applied Research in Intellectual Disability, 21 (6), 502-508.

Tsao, R. \& Kindelberger, C. (2009). Variability of cognitive development in children with Down syndrome: relevance of good reasons for using the cluster procedure. Research in Developmental Disabilities, 30 (3), 426-432.

Turner, S. \& Alborz, A. (2003). Academic attainments of children with Down's syndrome: a longitudinal study. British Journal of Educational Psychology, 73 (4), 563-583.

Vicari, S. (2006). Motor development and neuropsychological patterns in persons with Down syndrome. Behavior Genetics, 36 (3), 355-364.

Vygotsky, L. S. (1997). Los problemas fundamentales de la defectología contemporánea. In: Obras escogidas V: fundamentos de defectología. (pp. 1140). Madrid: Visor.

Wang, P. (1996). A neuropsychological profile of Down syndrome: cognitive skills and brain morphology. Mental Retardation and Developmental Disabilities Research Reviews, 2 (2), 102-108.

Wuo, A. S. (2007). A construção social da síndrome de Down. Cadernos de Psicopedagogia, 6 (11).

Ypsilanti, A. \& Grouios, G. (2008). Linguistic profile of individuals with Down syndrome: comparing the linguistic performance of three developmental disorders. Child Neuropsychology, 14 (2), 148-170. 